\title{
Wolchok Solid Tumors 2009 Oncology Response Criteria
}

National Cancer Institute

\section{Source}

National Cancer Institute. Wolchok Solid Tumors 2009 Oncology Response Criteria. NCI Thesaurus. Code C126051.

A measure proposed by Wolchok et al. (Clin Cancer Res 2009;15:7412-7420) to

determine the extent of an antitumor response to a particular immunotherapeutic agent. 